\title{
Artificial Olfaction Inside Nanostructured Infrared Fiber Arrays
}

\author{
Mecit Yaman ${ }^{1 *}$, Adem Yildirim ${ }^{1,2}$, Mehmet Bayindir ${ }^{1,2,3}$ \\ ${ }^{1}$ UNAM-National Nanotechnology Research Center, ${ }^{2}$ Institute of Materials Science and Nanotechnology, \\ ${ }^{3}$ Department of Physics, Bilkent University, Ankara 06800, Turkey \\ yaman@nano.org.tr
}

\begin{abstract}
Nanostructured hollow core fibers are used to demonstrate a new infrared absorption based artificial nose. The sensor unit of the array is a hollow core Bragg fiber that selectively guides incident blackbody radiation and enhances absorption for enhanced sensitivity.
\end{abstract}

Keywords; Bragg fiber, chemical sensing, artificial olfaction

\section{INTRODUCTION}

Artificial olfactory systems are designed for odour detection by means of an array of sensors used in conjunction with pattern recognition techniques. However, current artificial nose technologies are still only partially successful in one or two performance metrics in odour detection such as high selectivity and sensitivity, reusability, response time, interference from environment. Here we present a novel photonic nose device based on spectroscopic detection of odorous volatile organics using a hollow core photonic bandgap fibre (PBG) array (Fig. 1). The PBG fibre array is composed of specific infrared transmitting fibres [2] fabricated to match their transmission bands to the infrared absorption bands of a wide range of chemical groups, 400$5000 \mathrm{~cm}^{-1}$. Each odorant and/ or mixture is assigned a unique binary chemical code by observing signal quenching due to absorption, facilitating rapid and computerized odorant identification. Total number of chemical binary codes that can be addressed using $N$-fibre array is $2^{N}$, making it possible to address tens of thousands of chemicals using just 25 fibres. We expect that the system would find use in food/beverage quality inspection, pesticide detection and in disease diagnostics, health monitoring and pharmacokinetic studies.

The advent of photonic bandgap based hollow waveguides and fibres also enabled quantitative absorption spectroscopy in a miniaturized photonic bandgap gas cell with extended optical path lengths. In these hollow waveguides or fibres, micrometer-sized mode areas enable high beam intensities over centimetre to meter scale optical paths within a footprint area that is orders of magnitude smaller than conventional bulk cells. To date, trace level gas detection has been demonstrated with hollow waveguides as gas cells and lasers as infrared light sources for limited gaseous analytes, using metallic hollow waveguides with an external cavity QCL, using photonic crystal fibres (PCF) and for detection of gas analytes with p.p.m. to p.p.t. level concentrations. In these examples, however, hollow waveguides are used as simple waveguides/gas cells and spectral filtering is achieved by painstaking parameter tuning of the laser light sources. Alternatively, in a recent work, we showed that spatially variable photonic bandgap structures can be used as wavelength selectors with conventional gas cells as variable filter spectrometers [1]. We present a novel architecture where broadband light sources is used with wavelength scalable hollow fibre arrays both as wavelength selectors and gas cells for an wide sensing range (Fig. 1). Fibre array based infrared absorption sensor enables not only trace level detection, but also odour identification by using the combinatorial array response [2]. Additionally, in this scheme any chemical is given a "binary code" according to its infrared absorption pattern from an array of fibres.

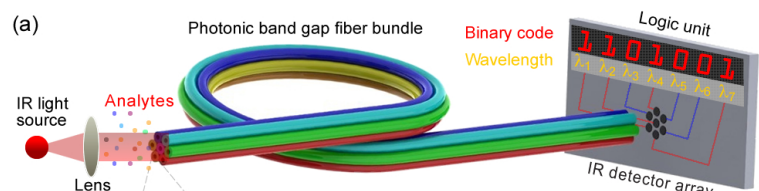

(b)

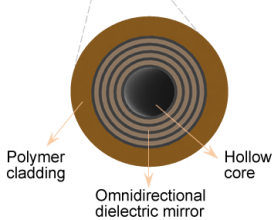

(c)

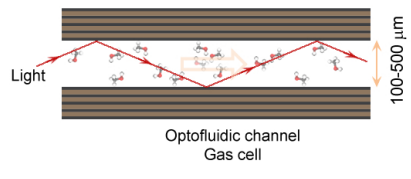

Figure 1 Schematic drawings of the digital fiber nose. (a) The sensor array consists of hollow core, infrared transmitting photonic bandgap fibers. Each fiber selectively guides a narrow band of the incoming continuum source. Spectrally filtered and guided radiation interacts with odorants in the hollow core of the fiber. The quenching of the transmitted intensity is recorded and registered as a binary code by comparing with a threshold value. (b) Nanostructured Bragg fiber for wavelength scalable waveguiding in the infrared spectrum (c) Optofluidic gas channel for infrared absorption spectroscopy. 

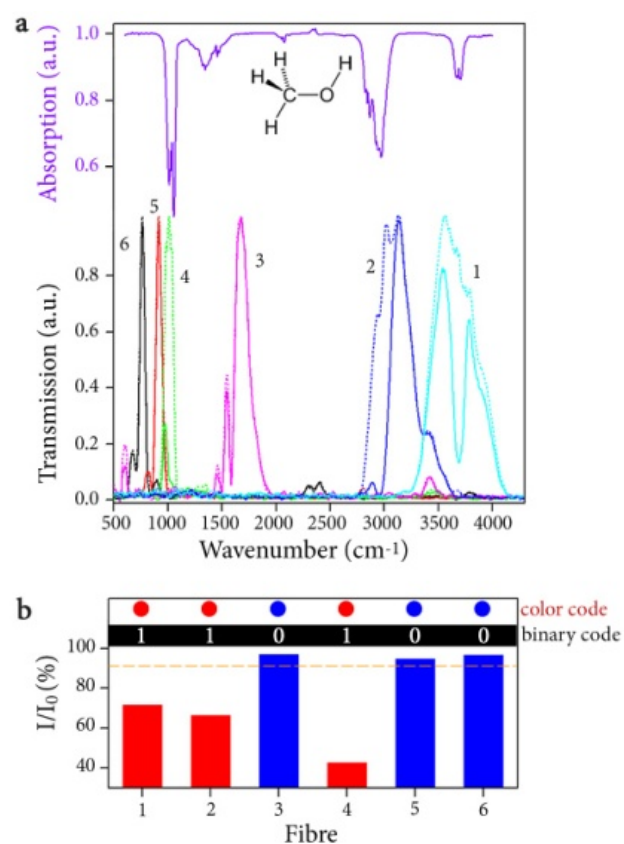

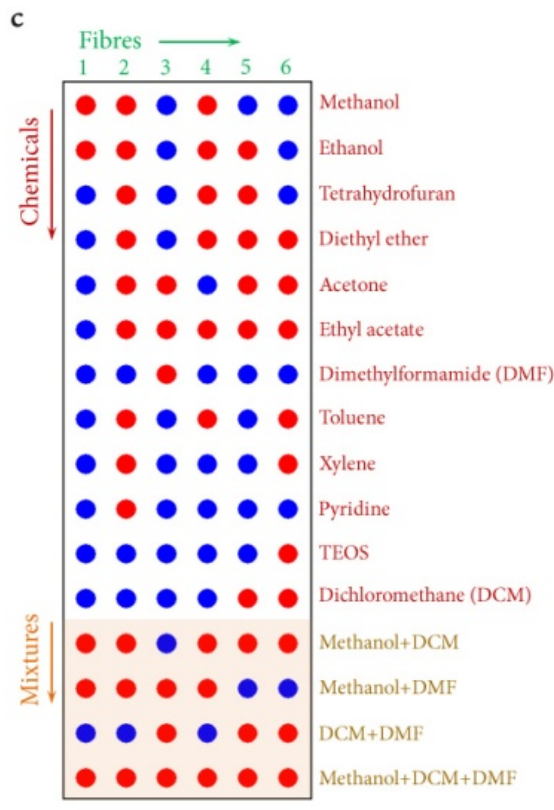

Figure 2. (a) Spectral intensity before (dashed) and after (solid) methanol introduction to the hollow core fiber. The decrease in transmission intensity after methanol introduction is shown to correspond to the methanol absorption bands. Peak $1058 \mathrm{~cm}^{-1}, 2976 \mathrm{~cm}^{-1}$ and $3688 \mathrm{~cm}^{-1}$ are detected by Fibre 4,2 and 1 , respectively. (b) The intensity ratio $\left(I / I_{0}\right)$ of each fibre in the array where $I_{0}$ is the transmission intensity and $I$ is the quenched transmission intensity. By taking a threshold value of $90 \%$, the fibre array response is represented using a binary number (blue for high intensity ratios, and red for low), resulting in a unique chemical code for each analyte. Binary code for methanol is determined to be "110 100". (c) A visual representation of twelve chemicals as seen by the photonic nose. Mixtures of three chemicals are also shown.

We use a broadband blackbody source as the infrared source together with a polymer/chalcogenide composite hollow core photonic bandgap fibre as both the waveguide/gas cell and the wavelength selector. These PBG fibres have periodic high index contrast dielectric multilayers inside its hollow core and therefore their transmission bands determined by the material refractive indices and quarter wave plate thickness, are structurally wavelength scalable and be specifically made to be in any part of the midinfrared regime simply by producing to smaller sizes during fibre drawing. Six distinct fibres with transmission bands spanning the mid infrared spectra, at 2.8, 3.3, 6.0, 9.9, 10.9 and $13.0 \mu \mathrm{m}$, are used as shown in Fig. 2a. The photonic bandgap transmission bands are fully or partially punctured with the intrinsic absorption of the polymer material due to the evanescent electromagnetic energy loss which penetrates through a number of Bragg layers.

Using the photonic nose, chemical signature of an analyte is obtained by measuring the fibre transmissions before and after the analyte introduction. The transmission intensities with and without the analyte is measured for each fibre and ac corresponding intensity ratio $\left(I / I_{0}\right)$ is calculated, where $I$ is the intensity with analyte in the fibre gas cell, and $I_{0}$ without. In Figure 2b, fibre array response to methanol vapour is shown.
By choosing an appropriate threshold value for $\left(I / I_{0}\right)$, the array response can be written as a binary code for any analyte. For example, a threshold value of $90 \%$ was taken and binary code for methanol is determined to be ' 110100 ', where each binary digit represents whether the intensity is above or below the threshold. In order to demonstrate the selectivity of the photonic nose, we chose twelve chemicals with wide range of chemical functionalities such as alcohols, ethers, aromatics, carbonyl containing molecules. The fibre array response for each analyte and ternary mixtures are visualized using a two-dimensional colour map (Fig. 2c). Furthermore, the fibre responses can easily be distinguished by converting the binary code to an equivalent decimal number.

\section{ACKNOWLEDGMENT}

This work was partially supported by State Planning Organization (DPT) and TUBITAK under the Project No. 106G090. M.B. acknowledges support from the Turkish Academy of Sciences Distinguished Young Scientist Award (TUBA GEBIP).

\section{REFERENCES}

[1] H. E. Kondakci, M. Yaman, M. Bayindir et al. Appl. Phys. Lett. 94, 111110 (2009).

[2] A. F. Abouraddy, M. Bayindir et al., Nature Mater. 6, 336 (2005).

[3] A. Yildirim, M. Vural, M. Yaman, M. Bayindir, Adv. Mater. 23, 1262 (2011). 